\title{
Evaluation of the Obstetrical Brachial Plexus Injuries with Forensic Perspective
}

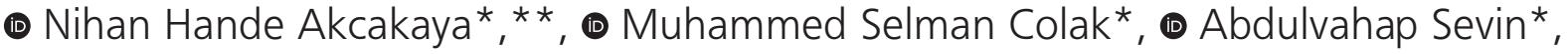

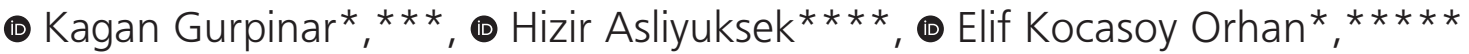 \\ ${ }^{*}$ Council of Forensic Medicine Istanbul, Turkey \\ **Demiroglu Bilim University Faculty of Medicine, Department of Neurology, Istanbul, Turkey

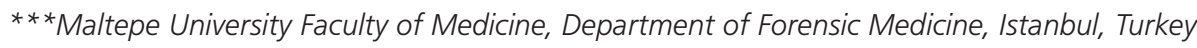 \\ $* * * *$ Istabul University-Cerrahpasa, Institute of Forensic Sciences and Legal Medicine, Istanbul, Turkey \\ $* * * * *$ Istanbul University-Istanbul Faculty of Medicine, Department of Neurology, Istanbul, Turkey
}

\section{Abstract}

Aim: Obstetrical brachial plexopathy (OBP) is the loss of function of the brachial plexus due to a traumatic, idiopathic, or iatrogenic reason since birth. OBP mostly occurs with a traumatic background that is secondary to a difficult birth. In this study, we aimed to investigate the medical characteristics of the OBP cases, which were evaluated for malpractice reasons by the Forensic Medicine and define the features that should be considered in the forensic evaluation of OBP.

Methods: The medical files of all cases with OBP evaluated in the $2^{\text {nd }}$ Specialization Board of the Council of Forensic Medicine between 2013 and 2017 due to malpractice were included in the study. The dates of birth, maternal ages, comorbid diseases of the mother, the number of pregnancies, birthplaces and birth weeks, birth weights, and additional comorbid pathologies were investigated retrospectively.

Results: A total of 287 cases were evaluated in this study. The mean age of forensic evaluation was found to be $2.9 \pm 2.3$ years. All patients were born with vaginal delivery except one caesarean case. In 55\% of cases had shoulder dystocia, 16\% clavicle fracture, $4 \%$ humerus fracture and humeral head dislocation were detected. $23.3 \%$ of the cases had a history of a difficult birth and $11.5 \%$ had assisted delivery. In $45 \%$ of cases had developmental pathologies accompanied by hypotonia. None of the cases were evaluated as medical malpractice.

Conclusion: Standard medical records of cases with OBPs contained sufficient information for neurological and forensic evaluation. Our study shows the OBP's does not only originate from the traumatic etiology; about half of the cases have different comorbid pathologies, especially neurodevelopment problems. In order to achieve standardization and understand the comorbidities of plexopathy better, it would be appropriate to expand the medical data in detail.

Keywords: Brachial plexus neuropathies, brachial plexus, parturition, obstetric, malpractice

\section{Introduction}

The brachial plexus is a complex peripheral neural network that provides innervation to the upper limb. The brachial plexopathies that occur with damage of this network lead to loss of muscle strength in the flask type of upper extremity. Pathologies seen in this neural network in newborns are called obstetrical brachial plexopathies (OBP). OBPs are rarely seen about 1-2 per 1,000 live births $(1,2)$. OBP can develop in the prenatal or intra-natal processes. The majority of OBPs are obstetric complications due to traumatic peri or intra-natal causes. Shoulder dystocia is the leading known risk factor (2). Other risk factors are high birth weight (macrosomia) and other comorbidities seen with birth trauma, such as assisted birth and breech presentation $(3,4)$.

Although OBP occurs due to the stress in the brachial plexus during childbirth, it can also be seen in cases that do not have any risk in the obstetric process and without any problems during the delivery (3). For instance, uncomplicated caesarean delivery is expected to decrease

Address for Correspondence: Nihan Hande Akcakaya, Counsil of Forensic Medicine Institute, 
the risk of OBP. However, OBP can also be seen in infants born with caesarean delivery. In these cases, maternal uterine malformation and familial obstetrical brachial plexus palsy may be considered among the prenatal processes. Besides, comorbid conditions such as hypotonic neonate or transient hypotonia of the newborn may be created by caesarean delivery, and perinatal asphyxia is considered another factor for OBP (3). It is interpreted that as a risk factor, hypotonia makes both the baby and plexus structures susceptible to stretching and creates a predisposition to brachial plexopathy $(3,5)$.

Brachial plexopathies are an important cause of disability in the young population, and improvement with treatment is very limited in this pathology $(2,6)$. Neurological evaluation and electrophysiological examination help understand the time of occurrence of the lesion and the type of involvement at the brachial plexus. Determining the kind of involvement is vital for planning the treatment $(5,7,8)$. In evaluating a newborn with OBP, accompanying conditions such as humerus or clavicle fracture, diaphragm paralysis should be considered. This approach should be carried out by a multidisciplinary team of pediatricians, neurologists, orthopedists, neurosurgeons, and physiotherapists. In this respect, OBP creates a financial and moral burden on families.

A large number of lawsuits are filed against OBP cases with malpractice allegations. When there is a medical malpractice claim to physicians or assisted health professionals, especially the course of pregnancy, the birth processes and the features that may predispose to plexopathy, such as macrosomia are investigated in detail with medical documents. In this study, we aimed to investigate the medical characteristics of the OBP cases evaluated for malpractice by the Council of Forensic Medicine, $2^{\text {nd }}$ Specialization Board and define the features that should be considered in the forensic evaluation of OBP.

\section{Methods}

In this study, all OBP cases with claims of malpractice brought to the $2^{\text {nd }}$ Specialization Board of the Council of Forensic Medicine between 2013 and 2017 were scanned from the medical files retrospectively with personal data protection. The Scientific Committee of the Forensic Medicine ethically approved this study (23.06.2017 with the number 21589509/256). Only the cases with signs of brachial plexopathy since birth were included in this study. All features of the detected cases were systematically noted and documented; i.e.; their birth dates, maternal ages, and diseases of the mother if any, how many pregnancies the mother had, who assisted the mother during the labor, birthplaces of the cases, birth weeks and weights, Electromyography (EMG) findings and applied treatments. In this retrospective study, descriptive statistical method was used as a statistical method. Basic statistical methods; mean value, percentage calculation and standard deviation were used to demonstrate the all the features of the data.

\section{Results}

A total of 287 OBP files were evaluated between 2013 and 2017, with an average of 57 per year (Figure 1). One hundred-fifty-six (54.3\%) of the cases were male and 131 (45.6\%) were female. According to file dates of the cases, the average age of forensic evaluation was determined as $2.9 \pm 2.3$ years (minimum: 0 and maximum: 17). The detailed evaluation of the files showing OBP was evaluated as a complication in all cases. Apart from one patient with caesarean birth, all of the other cases were born with normal delivery. Two hundred eighty-five babies were born in the hospital, and there was only one birth at home (Figure 2). In twenty-three cases out of 264, we were able to determine the health professional who assisted the delivery. In $77.3 \%$ (204) of the 264 cases, a physician-assisted the labor (Figure 3).

Among the 251 cases of which the birth weeks were detected; $89.6 \%$ of them were born in term (225), 5.6\% were preterm (19), and $2.4 \%$ were post-term (6). The

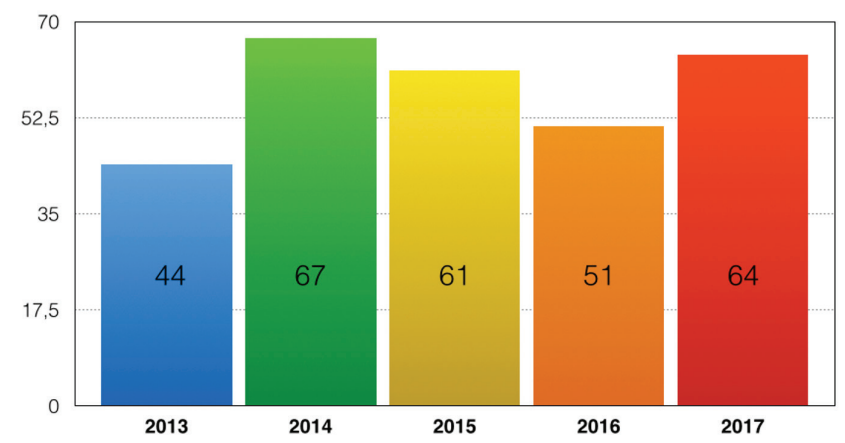

Figure 1. Distribution of the cases by years between 2013 and 2017

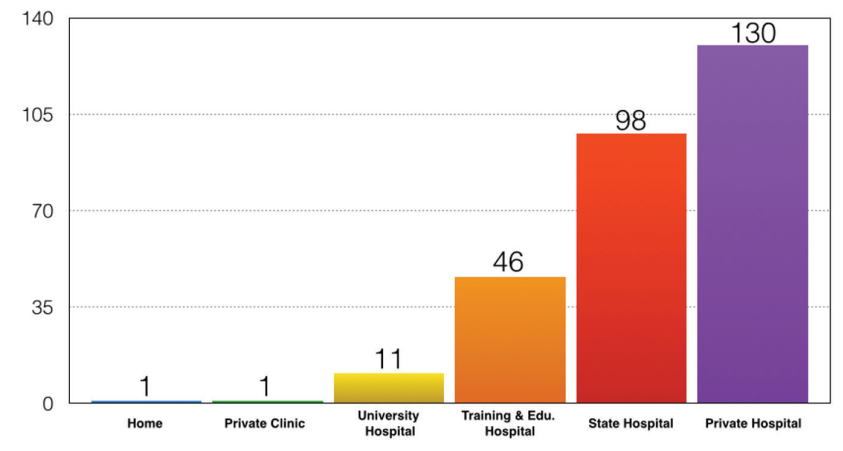

Figure 2. Distribution of birth places of the cases 
mean birth weight of the cases was $4.03 \pm 508.6 \mathrm{~kg}$ (Figure 4). There were 4 cases known to be born in the term but whose birth weight could not be determined from the file information. The frequency of the delivery presentation of the cases was cephalic (255), breech (2), and footling breech presentations (2). The delivery presentation could not be detected in 27 cases. Among the 175 mothers whose given birth age was seen, the average age was calculated as $29.7 \pm 5.6$ years. From the medical records, the medical history of 61 mothers were available. It was learned that 18 of the 61 mothers were healthy, 24 had diabetes, 8 had hypertension, 7 had obesity, and 3 had thyroid disease. Previous pregnancy information can be reached in 75 mothers, and 47 of them gave their first birth. Only four mothers had a history of a baby with plexopathy in her previous births. The EMG evaluation data and treatment of the cases were summarized in Figure 5.

Despite 25 cases that no information could be found about comorbid pathology in the files, the accompanying pathologies observed in OBP cases were very diverse. It was noted that there was more than one accompanying pathology in 138 of 262 cases. The most common accompanying pathology was found to be shoulder dystocia in 158 patients. The most frequently observed comorbid pathologies detected in the rest of 130 cases were patent ductus arteriosus, pulmonary hypertension, microcephaly, mental and motor developmental delay,

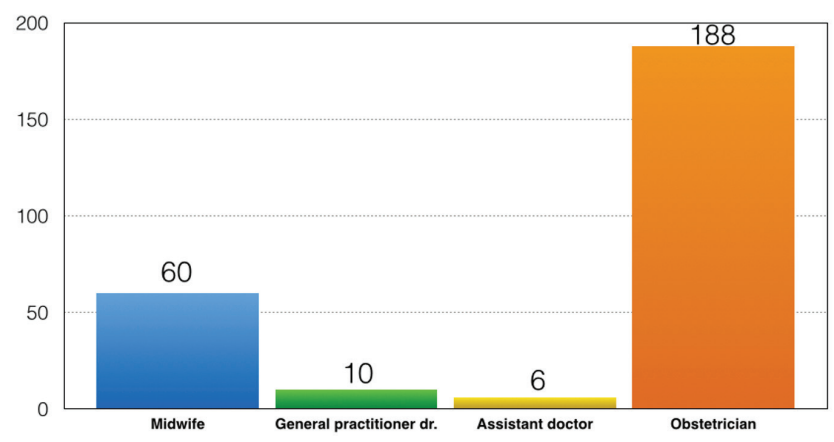

Figure 3. Distribution of health professionals for assisted delivery

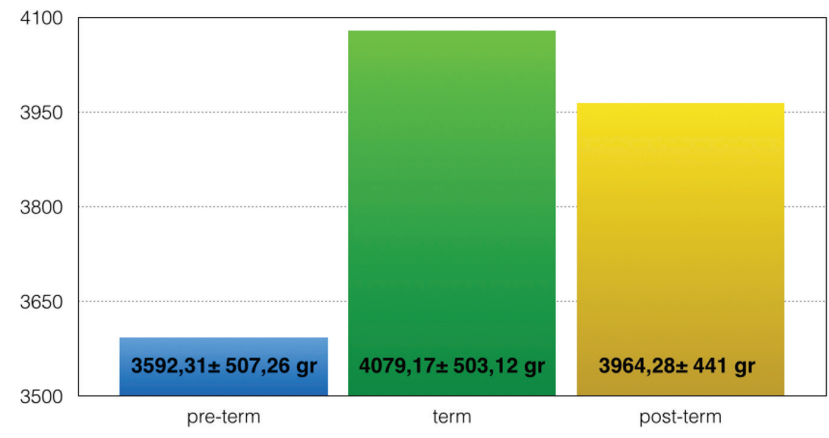

Figure 4. Average birth weight distribution by birth weeks

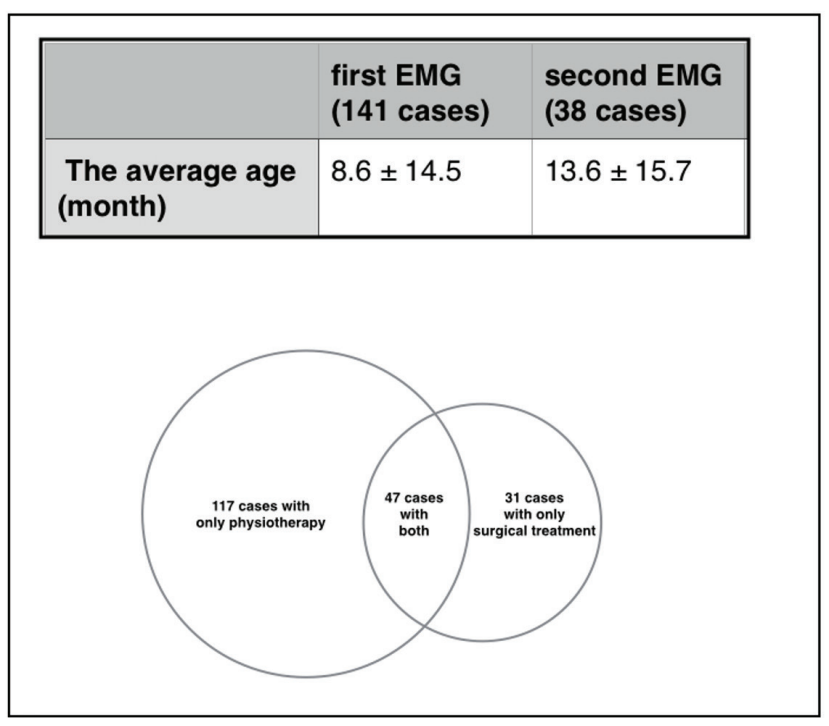

Figure 5. Age of EMG evaluation and treatment distribution of the cases. The average age of the first EMG study was calculated as $8.6 \pm 14.5$ months (min: 0 to max:108 months). Thirty-six patients underwent EMG study for the second time. The second EMG study's average age was $13.6 \pm 15.7$ months (min: 1 to max: 70 months). The 117 of 195 cases were treated with physical therapy only, 31 cases surgical only, and 47 cases both with physical therapy and surgically

hypotonia, oligo and polyhydramnios, neonatal convulsion, fetal bradycardia, hyperbilirubinemia, asphyxia, cyanosis, intracranial bleeding, anemia, infection, and caput succedaneum. There was clavicle fracture in 47 cases, humerus fracture or humeral head dislocation in 9 cases, and diaphragmatic paralysis in 3 cases. History of a difficult delivery, use of assisted delivery methods, prolonged labor, maternal insufficiency were noted in 67, 33, 9, and 2 cases, respectively.

\section{Discussion}

In this study, OBP cases on forensic evaluation within five years were systematically documented. The information about medical factors such as a history of difficult delivery, complications of delivery, shoulder dystocia, fetal macrosomia, gestational diabetes, material obesity, advanced maternal age, male fetus or post-term delivery, which are known to be associated with OBP in the literature, were investigated through the information from the medical documents. Traumatic damage of the brachial plexus during delivery may cause OBP, or it may also be seen for an undetectable reason (1,3,4,9-11). Therefore the damage at the plexus should be distinguished whether it is a result of traumatic obstetric complications or it is developed for a different reason. Complication management of the case is important where there are clues that OBP develops on a traumatic background, 
especially in complicated birth history, clavicle fracture. Examination notes, consultations, tests, and treatment of the case are essential for understanding complication management.

The most critical risk factor for OBP is stretching plexus structures during delivery $(3,4)$. Fetal macrosomia, abnormal birth presentation, difficult and prolonged delivery are important predisposing factors in $\operatorname{OBP}(9,10)$. Except for one case in our series, all of them were born in the hospital. Also, in all of them, childbirth was directed by trained healthcare professionals. In our series, there was no case under 2,500 $\mathrm{g}$ and 53 cases are macrosomic. It is noteworthy that the distribution of the average birth weight of preterm cases is $3592.3 \pm 507.2 \mathrm{gr}$. These findings once again point out the association of macrosomia with OBP (Figure 4).

In our series, the clues that OBP might derive from traumatic reasons and frequencies are shoulder dystocia in $55 \%$, clavicle fracture in $16.4 \%$, humerus fracture and humeral head dislocation in $4.2 \%$, history of difficult delivery in $23.3 \%$, assisted delivery methods in $11.5 \%$ of the cases. However, in 130 patients, who constituted $45 \%$ of the cases, there were different comorbid pathologies. These pathologies were patent ductus arteriosus, pulmonary hypertension, microcephaly, mental and motor developmental problems, hypotonia, oligo and polyhydramnios, and neonatal convulsion, which may indicate many comorbid heterogeneous different diseases. Our study showed that OBP might occur in healthy developing infants with traumatic background and as well as in cases with developmental problems. Although maternal and natal factors are discussed in the foreground in previous studies, it is predicted that OBP cases may be of genetic and intrauterine origin (9). In our study about half of the cases have neurodevelopmental problems supports literature. In about half of the cases, brachial plexopathy with developmental problems requires detailed further documentation and investigation.

When our series is examined in terms of documentation of the main predisposing factors that may cause OBP, it is seen that basic information such as birth weight, birth week, place of birth, and the assisted health professional of the delivery are well documented in almost all of the files. There are a few cases whose birth weight (1) assisted labor (23) and birth presentation (27) were not available. The cases are well documented in terms of basic predisposing factors. On the other hand, some basic information, such as the maternal factors known to pose a risk for $O B P$, is not well documented. In most of the files, mothers' comorbid diseases, how many births the mother had, or whether the mother has another child with plexopathy are not included. This situation is an obstacle in the standard evaluation of these cases.

In this study, in addition to the basic medical information of cases with OBP, EMG study and treatments were also documented in terms of complication management. In this respect, EMG information is not available in approximately half of the cases $(50.8 \%)$. In the remaining cases, there is great heterogeneity in terms of EMG study practice. Especially timing of EMG studies showed a wide range. This situation can be interpreted in terms of neurologists' having no standardization in the electrophysiological approach to OBPs (8). EMG should be considered as an extension of the neurological examination. The EMG study provides important information about the duration, spread, and degree of pathology. Therefore, EMG is important in terms of proper management of the cases and complication management. In our study, 110 of 141 cases who underwent EMG study were treated. It was also determined that 110 (57.6\%) of 191 patients referred to physiotherapy with or without plexus surgery had EMG. It is understood that further investigation with EMG study is associated with a high rate (78\%) of treatment.

Obstetrics and gynecology is the most common branch of all malpractice lawsuits in Turkey (12). It has been reported that psychosocial factors and lack of communication between the doctor and the family have an effect on malpractice lawsuits in which newborns are affected, rather than the severity of the involvement in the brachial plexus (13-15). For this reason, it is recommended that the obstetrician inform the family first-hand, that the involvement of the babies should be examined in detail with tests (especially EMG, standardized quality of life scales and functional upper limb scales) and detailed documentation of the comorbid factors including rare diseases of the infant $(14,16,17)$.

\section{Study Limitations}

The retrospective method is the major limitation of the study. Depending on the retrospective evaluation, it was not possible to reach the result of every parameter determined in every file. For instance, maternal medical history detected in \%21 (61/287) of the cases and this is the least informed parameter of the study. Despite the limitations in maternal medical information, the characteristics of the cases were well documented and our study revealed important results about characteristics of the OBP cases. The OBP cases with malpractice claim have been discussed for the first time in the literature. Our study will contribute to the literature with these features.

\section{Conclusion}

Etiopathology is a critical issue in cases that are evaluated for malpractice claims. The OBP has a 
multifactorial etiopathology. The OBP can occur as a comorbid to a developmental pathology as well as the well-known predisposing factors. This study also shows the OBP's does not only originate from the traumatic etiology; about half of the cases have different comorbid pathologies, especially neurodevelopment problems. Our study showed that the medical records of patients with OBPs contained sufficient information for neurological and forensic evaluation. However, to achieve standardization and to understand the comorbidities of plexopathy better, it would be appropriate to expand the medical data in detail.

\section{Authorship Contributions}

Concept: E.K.O., H.A., Design: E.K.O., N.H.A., Data Collection or Processing: M.S.C., A.S., Analysis or Interpretation: N.H.A., H.A., K.G., E.K.O., Literature Search: E.K.O., M.S.C., Writing: N.H.A.

Conflict of Interest: No conflict of interest was declared by the authors.

Financial Disclosure: The authors declared that this study received no financial support.

\section{References}

1. Coroneos CJ, Voineskos SH, Coroneos MK, et al. Primary nerve repair for obstetrical brachial plexus injury: a metaanalysis. Plast Reconstr Surg 2015;136:765-79.

2. Ünsal SS, Armangil M. Obstetrical brachial plexus palsy. Turk J Womens Health Neonatol 2020;2:47-52.

3. Heise CO, Gherpelli JLD. Prognostic relevance of risk factors for obstetrical brachial plexopathy. Arq Neuropsiquiatr 2006;64:30-4.

4. Foad SL, Mehlman CT, Ying J. The epidemiology of neonatal brachial plexus palsy in the United States. J Bone Joint Surg Am 2008;90:1258-64.

5. Yang LJS. Neonatal brachialplexus palsy, Management and prognostic factors. Semin Perinatol 2014;38:222-34.

6. Aras Y, Aydoseli A, Sabancı PA, Akcakaya MO, Alkır G, Imer M. Functional Outcomes After Treatment of Traumatic Brachial Plexus Injuries: Clinical Study. Ulus Travma Acil Cerrahi Derg 2013;19:521-8.

7. Spires MC, Leonard JA, Wolfe J. The role of electrodiagnosis in infants with brachial plexus palsies. In: Chung KC,
McGillicuddy JE, Yang LJ-S, editors. Practical Management of Adult and Pediatric Brachial Plexus Palsy. London: Elsevier; 2011. p. 68-74

8. Heise CO, Siqueira MG, Martins RS, Gherpelli JLD. Motor Nerve-Conduction Studies in Obstetric Brachial Plexopathy for a Selection of Patients with a Poor Outcome. J Bone Joint Surg Am 2009;91:1729-37.

9. Galbiatti JA, Cardoso FL, Galbiatti MGP. Obstetric Paralysis: Who is to blame? A systematic literature review. Rev Bras Ortop 2020;55:139-46.

10. Avram CM, Garg B, Skeith AE, Caughey AB. Maternal bodymass-index and neonatal brachial plexus palsy in a California Cohort. J Matern Fetal Neonatal Med 2021:1-8.

11. Vakhshori V, Bouz GJ, Alluri RK, Stevanovic M, Ghiassi A, Lightdale N. Risk factors associated with neonatal brachial plexus palsy in the United States. J Pediatr Orthop B 2020;29:392-8.

12. Büken $E$, Büken NÖ, Büken B. Obstetric and gynecologic malpractice in Turkey: incidence, impact, causes and prevention. J Clin Forensic Med 2004;11:233-47.

13. Zaami S, Busardò FP, Signore F, et al. Obstetric brachial plexus palsy: a population-based retrospective case-control study and medicolegal considerations. J Matern Fetal Neonatal Med 2008;31:1412-7.

14. McAbee GN, Ciervo C. Medical and legal issues related to brachial plexus injuries in neonates. J Am Osteopath Assoc 2006;106:209-12.

15. Abzung JM. Psychosocial Factors Are an Important Aspect of Parent and Patient-Reported Outcomes in Neonatal Brachial Plexus Palsy: Commentary on an article by Emily A. Eismann, MS, et al.: "The relationship between medical malpractice litigation and parent reports of patient function following neonatal brachial plexus palsy". J Bone Joint Surg Am 2014;96:43.

16. Eismann EA, Bauer A, Kozin SH, Louden E, Cornwall R. The relationship between medical malpractice litigation and parent reports of patient function following neonatal brachial plexus palsy J Bone Joint Surg Am 2014;96:373-9.

17. Medeiros DL, Agostinho NB, Mochizuki L, Oliveira AS. Quality of life and upper limb function of children with neonatal brachial plexus palsy. Rev Paul Pediatr 2020;38:e2018304. 